\title{
Active Electronically Steered Array (AESA) Radar Data Visualization Software
}

\author{
K. Kavitha ${ }^{1}$, E. Srinivas Reddy ${ }^{2}$ and Dr. N.V Rao ${ }^{3}$ \\ ${ }^{1}$ Asst. Professor, CVR College of Engineering/IT Department, Hyderabad, India \\ Email: kavithablessyita@gmail.com \\ ${ }^{2}$ Scientist- 'D', RCI (Research Centre Imarat)/DRDO, Hyderabad, India \\ Email: reddyesr1346@rediffmail.com \\ ${ }^{3}$ Professor, CVR College of Engineering/CSE Department, Hyderabad, India \\ Email: nvr@ieee.org
}

\begin{abstract}
AESA stands for Active Electronically Steered Array based Antenna. In this type of Radar, the antenna is viewed as a two-dimensional array of radiating elements. Number of radiating elements varies from few hundreds to few thousands. Each radiating element is illuminated with an underlying Transmit-Receive Module (TRM). The AESA system is controlled by Radar Controller Software designed with required GUI controls for accepting data from operator.

The proposed Radar Data Visualization Software integrates into Radar Controller Software as an independent module. It uses AESA Radar data that is being handled in Radar Controller Software and presents the data to the operator in wide variety of forms including File view, Tree View, Table view, Beam view etc.,
\end{abstract}

Index Terms: TRM (Transmit and Receive Module), SPI, Radar Data, Elevation Angle, Azimuth Angles, Views, Antenna.

\section{INTRODUCTION}

The RADAR system is used for detecting an object by using electromagnetic waves; it also identifies the range, the altitude, the direction and the speed of both the moving and the stationary objects. It also does searching and tracking of aerial objects by positioning the RADAR beam at desired angles. The RADAR acronym is Radio Detection And Ranging, it was coined in 1940 by the U.S Navy. The applications of radar are aircraft, ships, motor vehicles, weather formations, terrain etc.,

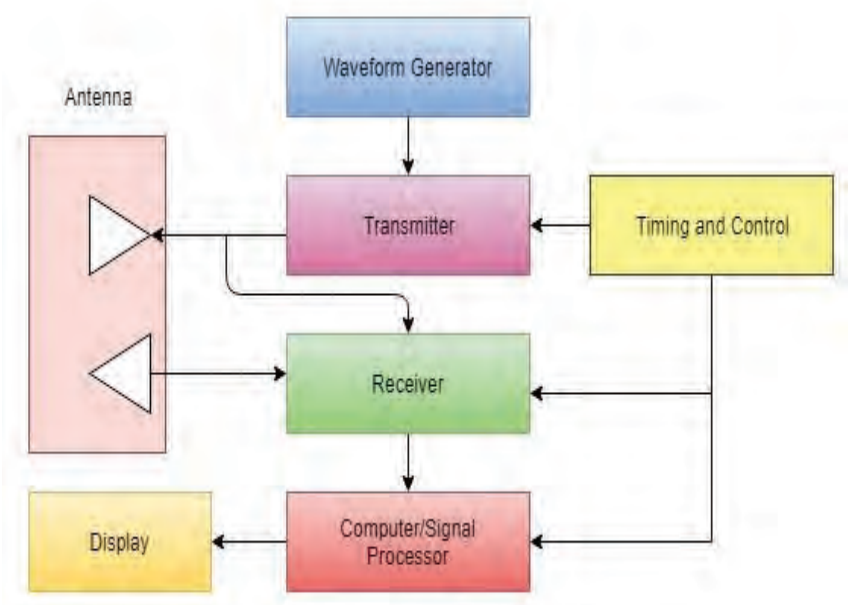

Figure 1. Basic principle block diagram of Radar
Conventional Radars do have a gimbal based mechanism for steering the beam from one position to another position. Generally, "Fig. 1" shows the Radar systems do work by connecting an antenna to a radio transmitter which is very powerful in nature to transmit a short pulse of signal. Then transmitter is disconnected and the antenna is attached to a sensitive receiver which intensifies any reverberation from target objects and then sends the resulting output to a display and the transmitter elements are typically klystron tubes, which are suitable for amplifying a small range of densities.

In order to examine a portion of the sky, the radar antenna has to be physically rotated to point in different directions. New trend in RADARs is Electronically Steering the beam position, called as AESA Radars. In AESA Radars, single transmitting source is replaced with many typically few hundreds of Transmit-Receive Modules.

This paper describes AESA Radar Data Visualization Software which is a real time application developed using QT Designer. The following section II deals with technical background of the AESA Radar which may be necessary to understand the terminology of each electronic system used in the project. Working of AESA Radar Controller Software and communication between all the Controllers are discussed in section III.

\section{TECHNICAL BACKGROUND}

Terminology of Radar:

Radar can be classified into many ways[1]:

1. Whereabouts of Radar: Ground based, Ship borne, Airborne, Space borne.

2. Actions performed by Radar: Tracking, Surveillance, imaging, data link, exploration.

3. Radar utilization: air traffic control (terminal area), air defense (application to defense), monitoring of surface (remote sensing), space borne measurement of sea surface, ground penetrating radar (geology).

4. Frequency band

5. Radar Beam Scanning: Fixed beam, electronic scan (frequency control and mixed in azimuth/elevation), mechanical scan (rotating, oscillating), multi beam configurations.

6. Different data accessed from Radar: Range (delay time), azimuth (antenna beam pointing for both 
horizontal and vertical), power (amplitude), and elevation (3-D functional tracking for both horizontal and vertical), and speed (measurement of different phase due to Doppler Effect coherency).

7. Radar contour: mono-static (transmit and receive same antenna), bi-static (separated transmitter and receiver - two antennas), multi-static (one or more transmitters/receivers).

8. Transmitter waveform: continuous, pulsed and digital.

9. Hardware machinery used for radar: antenna, array, transmitter, receiver.

\section{Transmitter:}

Transmitter can also be referred as an oscillator, which is also pulsed by the modulator to generate trains of pulses.

The transmission line helps to channelize the waveform to the antenna which is then emitted into the space. One antenna can perform the function of both the receiver and the transmitter.

\section{Receiver:}

The receiver is of the super heterodyne type. Low noise RF amplifier is the first stage, such as parametric amplifier or a low noise transistor.

The input is fed into the device at the mixer stage, in spite of a receiver with a low noise. The front end will be more sensitive and this mixer input can have a high dynamic range, less sensitivity to overload and less compulsion to electronic interference.

\section{Antenna:}

The tracking, processing and successive measurement of the estimated target are done by the radar antenna. The radar antenna is the common form of reflectors that is of a parabolic shape.

The beam is scanned in space by using a mechanical pointing mechanism of the antenna. Imaging can also be done by generating two or three dimensional image of targets. Classification, discrimination and identification, determining the characteristics, type and identity of a target are also done by the radar.

\section{Formation of Beam:}

Beam forming [3] is crux, that the constructive sum is a set of signals emitted from a set of small non-directional antennas in order to form a large directional signal. Once the signal is generated it can either be physically steered by pointing the antenna in the particular direction, or it can be electronically steered without disturbing it mechanically.

By having a clear signal at the receiver, it allows the beam steering to have a precise method for signal's maximum power output to reach its destination. All individual elements of the antenna array will radiate the waves with different amplitudes and phases. Due to this difference in amplitude and phase these waves conflict with each other both constructively (causing peaks) and destructively (causing nulls) for designing the overall radiation pattern for the full array.

\section{AESA RADAR}

Active Electronically Steered Array (AESA) radar takes an approach of using an array antenna into a further step. Instead of shifting the phase signals from a single high power transmitter here, AESA employs a grid of hundreds of small "Transmitter-Receiver (TR)" Modules that are associated together by high-speed processors.

TR Modules are arranged in a row that forms a Linear Array (LA) which is shown in "Fig. 2". Associated with each linear array there is a Linear Array Controller (LAC) unit. This LAC controls all the TRMs in the linear array this is stacked upon each other to form a complete twodimensional array of radiating elements. A high-speed digital system called as an Array Controller (AC) unit is used to control the Linear Array Controller units in the array.

Radar Controller (RC) controls the operation of the AESA Radar in terms of calibration mode, operation mode etc., by commanding the Array Controller unit with appropriate data.

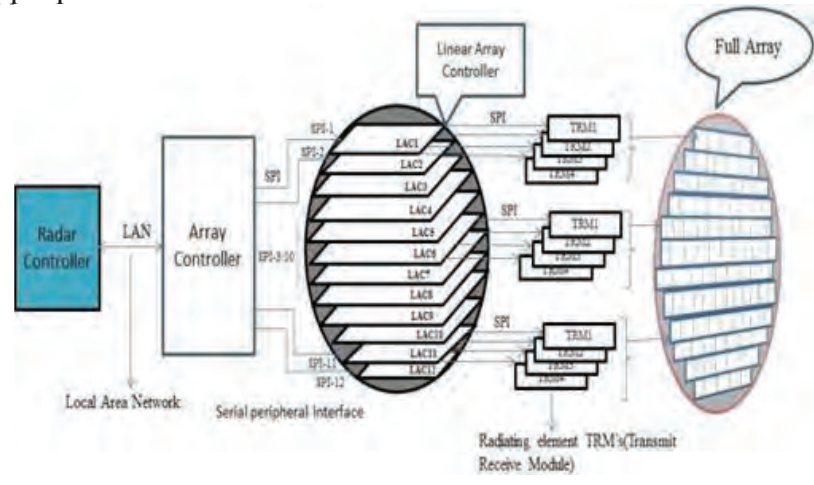

Figure 2. Block Diagram of AESA Radar

The AESA radar will radiate a multiple beams of radio waves at different frequencies simultaneously. The emission of AESA radar signals across a wider range of frequencies, which it makes them more over difficult to detect background noise. The ships and aircrafts may radiate powerful radar signals while still they remain stealthy. Most of the radar systems used in modern combat aircrafts is AESA systems.

Each TR module has its own transmitter, receiver, processing power and small spike like radiator antenna on top. This TR module has been computed to act as a transmitter; receiver and TR modules can be assigned to any role with output power or receiver sensitivity of any one of the "subsystems".

\section{A. Controllers}

\section{Radar Controller :}

The Radar Controller is connected to the array controller over LAN (Local Area Network) /Ethernet on this interface, the interaction happens between both of them is server-client fashion using TCP/IP protocol. The radar controller acts as a server and array controller as a client. This radar controller issues command, array controller executes that command and sends back response. 


\section{Array Controller:}

The Array Controller interacts with the Linear Array Controller units on individual, dedicated SPI (Serial Peripheral Interface) channels. In this context, the Array controller acts as Master and Linear array Controller as slave over the SPI channels, which are capable of operating in full-duplex mode, with 50 MBPS data rate.

\section{Linear Array Controller:}

Each array has got a controller unit, called as Linear Array Controller (LAC). The linear array controller interacts with its TRMs on individual/dedicated SPI channels, linear array controller acts as Master and TRM acts as Slaves. The SPI channels are capable of operating in simplex mode (i.e., data transfer happens from linear array controller to TRM) with $10 \mathrm{mbps}$ data rate.

\section{Transmit-Receive Module (TRM):}

TRMs known as Radiating Elements are arranged in Linear Array Controller. Each TRM will have a set of programmable values namely Tx Phase, Tx Attenuation, Rx Phase, Rx Attenuation to control the element and array beam. So, when compared to conventional RADAR, AESA radar has to handle huge data.

\section{B. Communication Interface}

SPI (Serial Peripheral Interface) is a synchronous protocol which is defined as clock, data and latch combination for data transmission from Array Controller to individual TRM. Devices communicating via SPI are in a master-slave relationship.

In "Fig. 3" SPI [2] is a single master communication protocol. It means that only one central device initiates all the transmissions with the regarding slaves. In "Fig. 4" it shows how the SPI master communication wants to send data to a slave and/or request information from it. This will select a slave by pulling the associated SS line low, and it activates the clock signal at a clock frequency which is being used by the master and the slave.

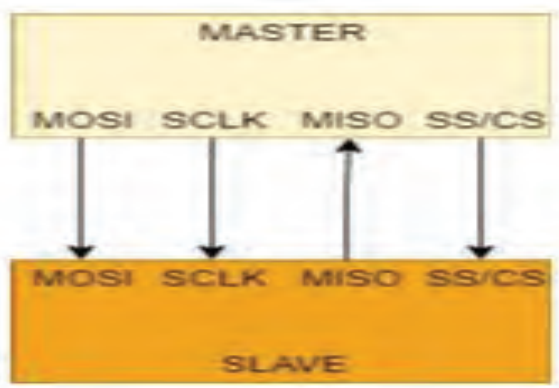

Figure 3. SPI Block diagram

A master/slave in "Fig. 5" show how the same set of slave parameters are used to communicate with the same set of master parameters. If the multiple slaves are used to connect to the master then it will have the different fixed configuration parameters.

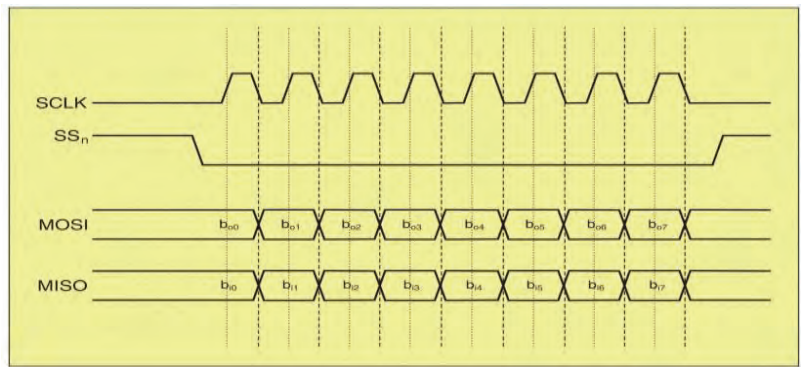

Figure 4. A simple SPI communication

Master Slaves:

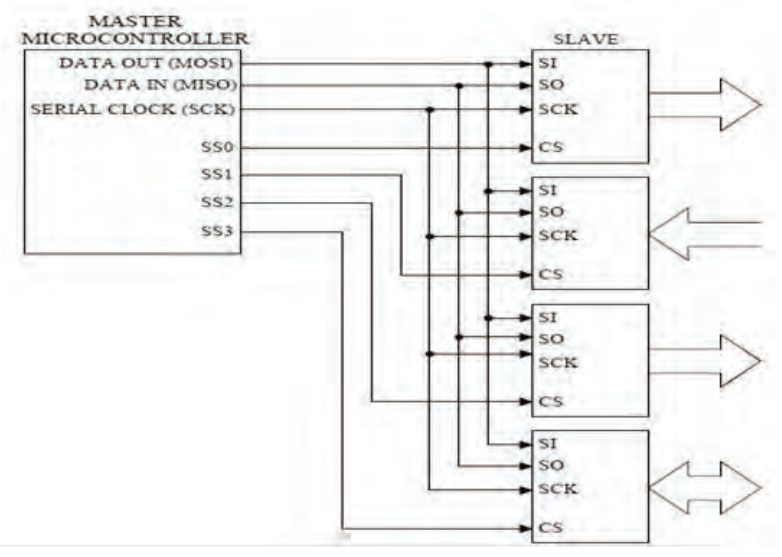

Figure 5. Block diagram of Multiple slaves

\section{Work Flow of SPI:}

SPI works in a "synchronous" data bus, it means that this data bus uses separate lines for "data" and a "clock", which keeps both in perfect synchronization.

\section{Sending Data:}

The clock which is a data bus and also it acts as an oscillating signal that it tells the receiver exactly when to send the sample bits on the data line, so that it will be the rising or falling edge of the clock signal. "Fig. 6" shows how the receiver detects that edge; it will immediately look at the data line to read the next bit. Along with the data the clock is sent, to specify the speed, though devices can be operated at the top speed.
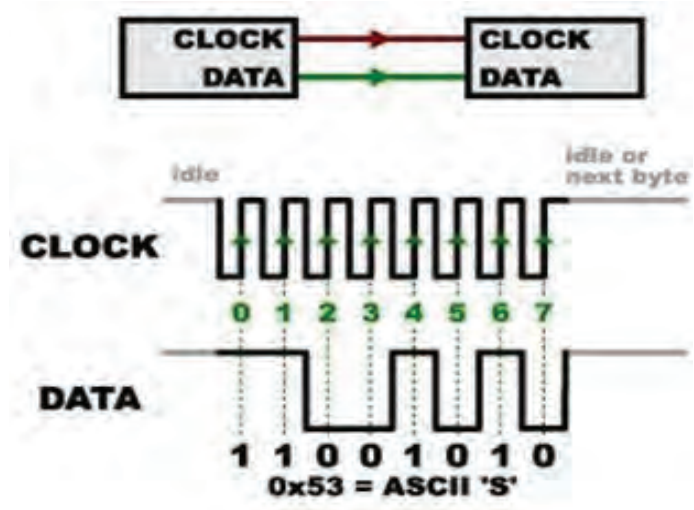

Figure 6. Sending data 


\section{Receiving Data:}

In SPI, it generates the clock signal (called as CLK or SCK for Serial Clock). The clock side which is generated is called the "master" and another side which is generated is called the "slave". In SPI, only one master (which acts as microcontroller) and also it can have multiple slaves.

In "Fig. 7" it shows how the data is sending from the master to a slave, this data is sended on a data line called MOSI (Master Out / Slave In). The response from the slave sent back to the master, then the master will continue to generate a prearranged number of clock cycles, and the slave will put the data onto a third data line called MISO (Master In / Slave Out).
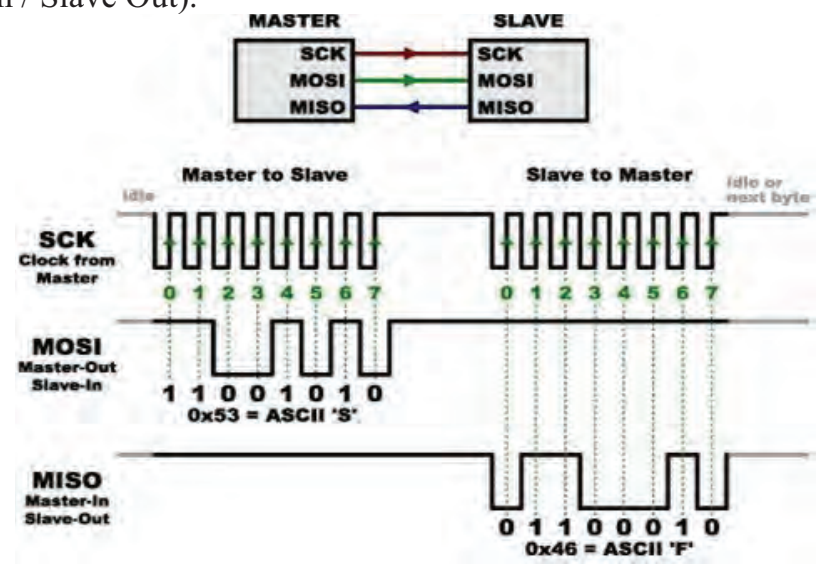

Figure 7. Receiving data

\section{Slave Select Working:}

The "Fig. 8" Slave Select is also called SS, it tells that it should be active to receive or send data and be present in multiple slaves to select each slave chip. The SS line is normally held high, which disconnects the slave from the SPI bus.
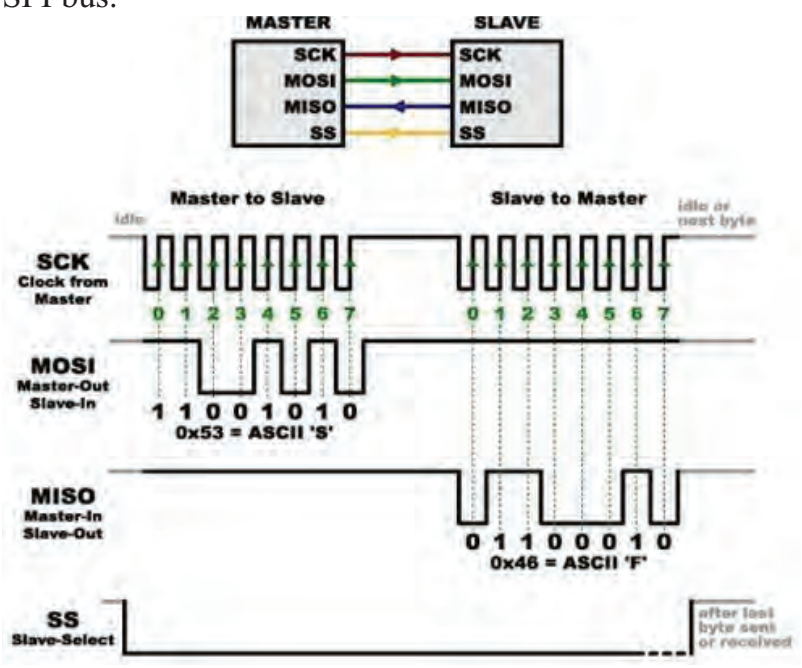

Figure 8. Working of Slave Select

\section{SPI Capabilities:}

Full-Duplex (Communicates in both directions simultaneously), multiple Mbps transmission speed of 0-50 $\mathrm{MHz}$ clock speed and transfers data in 4-16 bit characters.

\section{SPI Bus Wiring:}

SPI bus wiring is done like shown below:

Master-Out, Slave-In (MOSI),

Master-In, Slave-Out (MISO),

System Clock (SCLK),

Slave Select / Chip Select (SS1, SS or CS).

SPI Signal Functions:

MOSI - carries data out of master to slave

MISO - carries data out of slave to master (both MOSI and MISO are active during every transmission)

SS\# (or CS) - unique line to select each slave chip

SCLK - produced by master to synchronize transfers

\section{DESIGN AND IMPLEMENTATION}

\section{A. Developing of GUI for Radar Controller}

In "Fig. 9" it talks about the process flow of entire application how it is working.

\section{Communication with Array Controller:}

The interaction between Radar Controller and Array Controller happens in server-client fashion using TCP/IP protocol. $\mathrm{RC}$ acts as server and $\mathrm{AC}$ as client, $\mathrm{RC}$ issues commands, $\mathrm{AC}$ will executes them and sends back the response.

2. Interface Messages between Radar and Array Controllers:

\section{Health:}

Operator will initiate the health checking of LAC(s) or complete array by clicking a button on GUI. RC will issue a health command to AC. AC in-turn issue the command to selected LAC(s). LACs will respond back to AC. AC will post the response to RC. After due processing of response message, $\mathrm{RC}$ will display the individual TRMs health status on GUI.

Signature:

The Signature command (message) is used for knowing the hardware batch numbers, software version no. and checksum of Linear Array.

Status:

The status command (message) is to read-back the programmable values that are set to TRMs.

Programmable values:

The programmable values ( $\mathrm{Tx} \mathrm{Ph}$ (Transmit Phase), Rx $\mathrm{Ph}$ (Receive Phase) and Rx Attn (Receive Attenuation)) uses total 8-bits, 2-bits are used for identifying and remaining 6-bits are used for sending the data.

Each LA (Linear Array) uses 32db power supply for whole the TRM's inside it, means each TRM in the linear array will use $0.5 \mathrm{db}$ power. Attenuation is used to decrease the power of each TRM in the linear array and phase is used for moving radar antenna, in degrees like $0^{\circ}, 45^{\circ}, 67.5^{\circ}, 90^{\circ}$ up to $360^{\circ}$.

\section{CW Mode:}

Each TRM is tested in CW mode only, to get the absolute amplitude and phase values in VNA (Virtual Network Analyzer) 


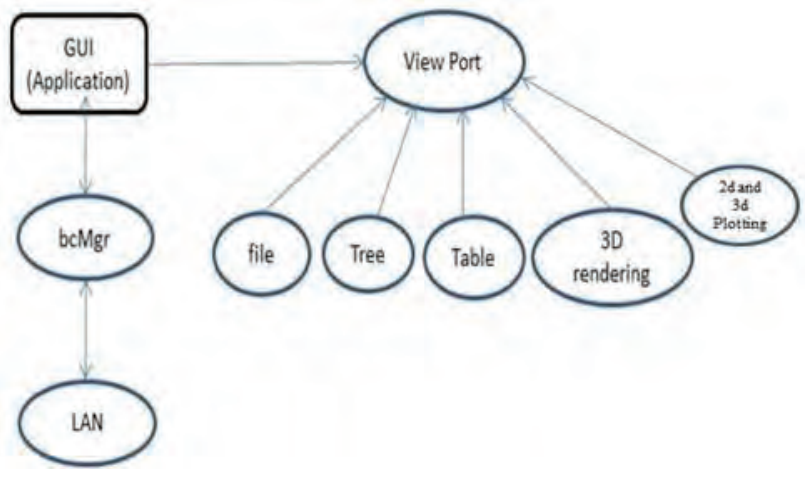

Figure 9. Process flow of entire application

\section{Data representation to the user:}

\section{File view:}

This File View is used to store the programmable values in the text file, so that any time modifications can be done and can import, load and share the file. The QFile System Model class provides a data model for the local file system in the file view.

The fileInfo (), isDir (), name () and path () functions provide information about the files and directories related to items in the model. Directories can be created and removed using mkdir (), rmdir ().

QFileSystemModel :: QFileSystemModel (QObject * parent $=0)$

QFileSystemModel *model = new

QFileSystemModel;

Model -> setRootPath (QDir::currentPath ());

\section{Table view:}

The Table View is used to show the values in the 2D table as rows and columns so that any calculations required for the element can be done easily. The QTableView class is one of the Model/View Classes and is a part of Qt model/view framework.

A QTableView implements a table view that displays items from a model. This class is used to provide standard tables that were previously provided by the QTable class, but more flexibility is provided by Qt's model/view architecture.

QTableView::QTableView (QWidget * parent = nullptr)

\section{Tree view:}

The TreeView is an hierarchical structure of radar controller, linear array controller and TRM values. In this structure the values of particular radiating element can be shown clearly. The QTree View class provides a default model/view implementation of a tree view.

A QTree View implements a tree representation of items from a model. This class is used to provide standard hierarchical lists that were previously provided by the QList View class, but more flexible approach is provided by Qt's model/view architecture.

\author{
QTreeView::QTreeView (QWidget * parent $=0$ ) \\ QFileSystemModel *model = new \\ QFileSystemModel; \\ Model->setRootPath (QDir:: currentPath ()); \\ $\underline{\text { QTreeView }}$ *tree $=$ new $\underline{\text { QTreeView }}$ (splitter); \\ tree->setmodel (model);
}

\section{Beam View:}

The rendering of data surface is done through the OpenGL using 3D graphics so that the beam position can be seen clearly. A beam formed out of the values, is shown as a $3 \mathrm{D}$ projection to mimic the real-world scenario.

The beam steering is animated while the AESA RADAR steers its beam according to its operation. This Beam View is done through the Qt OpenGL, with the QGL library file. In profile "QT $+=$ opengl" should be added. Using QGLWidget the OpenGL functions can be used. The main functions that are used for producing a 3D graphics of beam are the following,

Void initializeGL

Void resizeGL ()

Void paintGL ()

\section{Plotting:}

In this work, 2D plotting is done for Rx Phase and $\mathrm{Rx}$ Attenuation values separately using 'plot' command for 2D projection and setting $\mathrm{x}$ and $\mathrm{y}$ ranges according to the TRM values which are drawn from the measurement data. 3D plotting is done for phase and attenuation values of $\mathrm{Rx}$ and projected in 3D using 'splot' command through terminal based on both phase and attenuation values from the data file.

Number of plotting styles is there in Gnuplot for plotting $2 \mathrm{D}$ or $3 \mathrm{D}$ plots. Few commands like set style data and style function change the default plotting style for subsequent 2(D) 'plot' and 3(D) 'splot'. Each plot has its own expected set of data entries in a data file. The illusion of $3 \mathrm{D}$ is enhanced by choosing hidden line removal or depth-sorted surface elements. The 'splot' command can also calculate and draw contour lines corresponding to contour $\mathrm{z}$ values. A special case of 'splot' command is to map the $\mathrm{z}$ coordinate onto a $2 \mathrm{D}$ surface by projecting the plot along with $\mathrm{z}$-axis.

\section{B. Beam Display in $3 D$}

In "Fig. 10" the three-dimensional-(3D) Beam [4] is constructed in OpenGL in accordance with vector graphics rules. Every object is composed of a certain number of polygons. The most essential problem is arrangement of the objects in order starting from those, lying at the farthest, up to these, which are located nearest to the observer. OpenGL offers z-Buffer algorithm to perform this assignment. It uses a structure of the memory, where $Z$ coordinates of every pixel are stored. For each animation frame, $\mathrm{Z}$ coordinates of all the pixels, which constitute the $3 \mathrm{D}$ objects display, are compared with $\mathrm{Z}$ coordinates saved in Z-Buffer (of the previous frame). In case a specific pixel is characterized with shorter $\mathrm{Z}$ coordinate, then it is drawn up its previous place. 


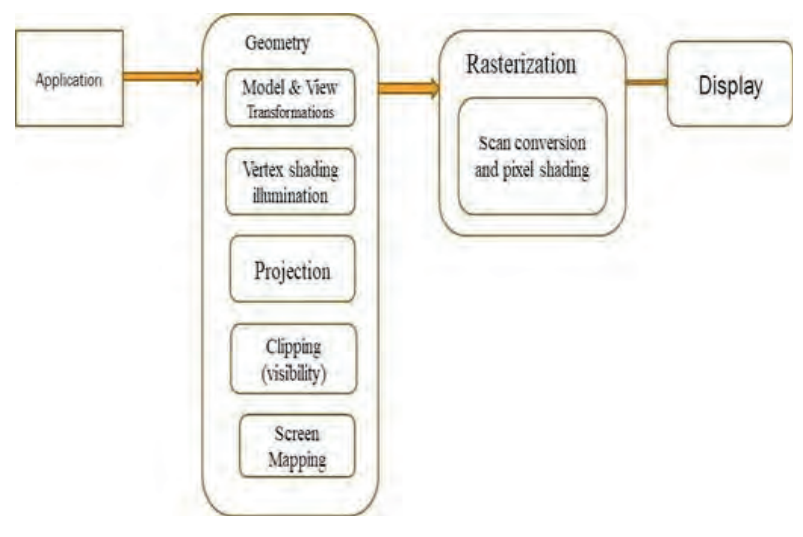

Figure 10. Process flow of beam in 3D

The next step in visualization of $3 \mathrm{D}$ stage is texture mapping. It consists in covering an object composed of 3D polygons, with a two-dimensional bitmap. Textures placing algorithm is based on at least two bitmaps, different in sizes. If the observer is close to the object, then the largest bitmap is projected on the beam. With its movement, the bitmaps are replaced each time with smaller ones.

\section{Estimation of Elevation and Azimuth angles}

Radar systems have become available for automotive, surveillance, anti-drone, medical and personal security applications. Due to this the need of inexpensive high performance radar is increasing. FWMC (Frequency Modulated Continuous Waveform) radar [6] has become popular due to its inheriting ability to utilize a large bandwidth with a low sampling rate.

The FMCW radar is one of the most popular $\mathrm{CW}$ radar types with ability to obtain range information. A K-band portable radar with beam forming [7] array is used to localize short range. It includes both transmitter and receiver channels. The beam forming array is a linear array element in which each element is a series-fed micro strip patch array element. The beam of the array can be continuously steered with a range of 45 degrees on the H-plane through an array of radar controllers.

\section{Effective beam pattern}

The effective beam pattern $[8,5]$ is derived from the radar data analysis and radar phase optimized values. The height of the melting layer is determined from range-height indicator (RHI) that scans from the radar bright band signature by considering only the single beam pattern. The pattern was measured from the isotropic radiated power and normalized by the calculated transmit power given by the RF system output power.

\section{E. Experimental Results}

Based on the QT software the below shown application is developed. The "Fig. 11" is the output design of entire radar application and it shows how each module is connected.

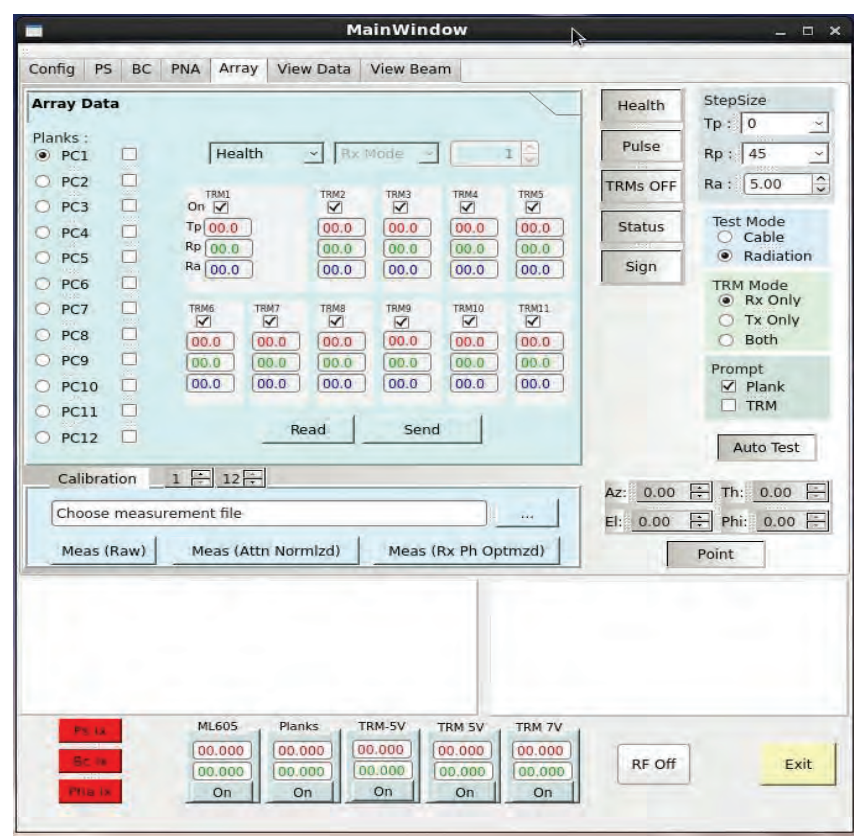

Figure 11. Design of Radar Application Software

The below shown "Fig. 12" is the output of some random values generated from the AESA radar in table view.

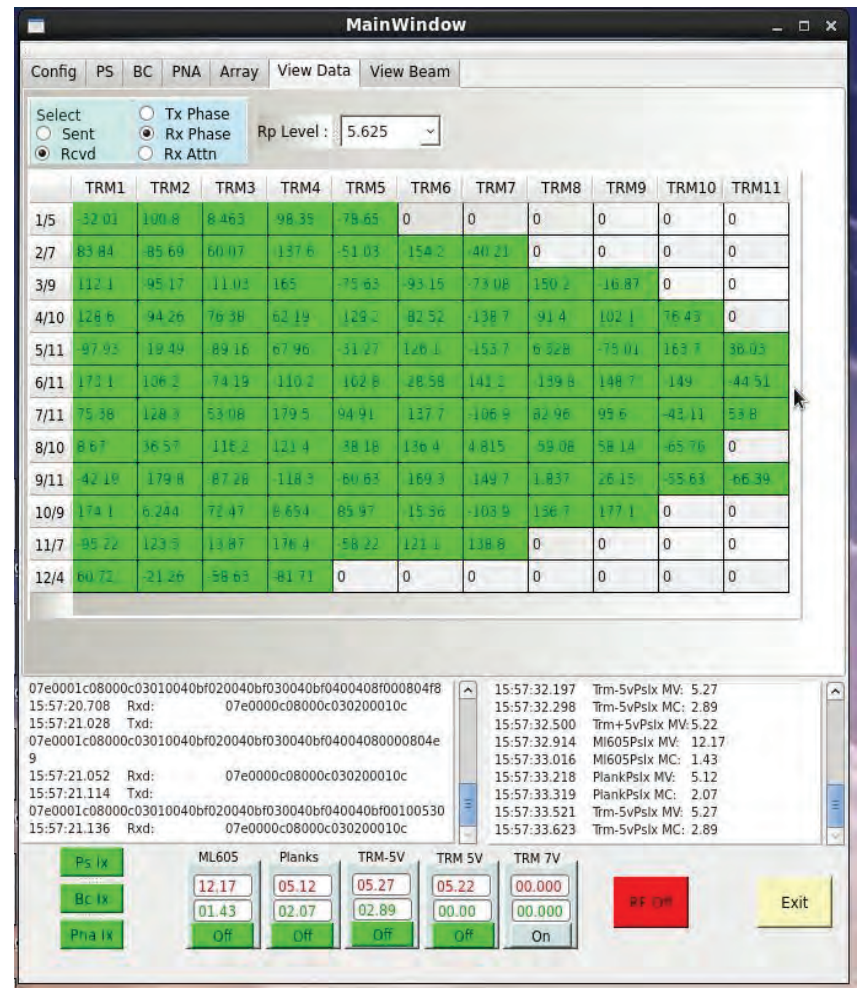

Figure 12. Data viewed in Table view

The below shown "Fig. 13" is output of beam steering at desired angles shown in projection view 


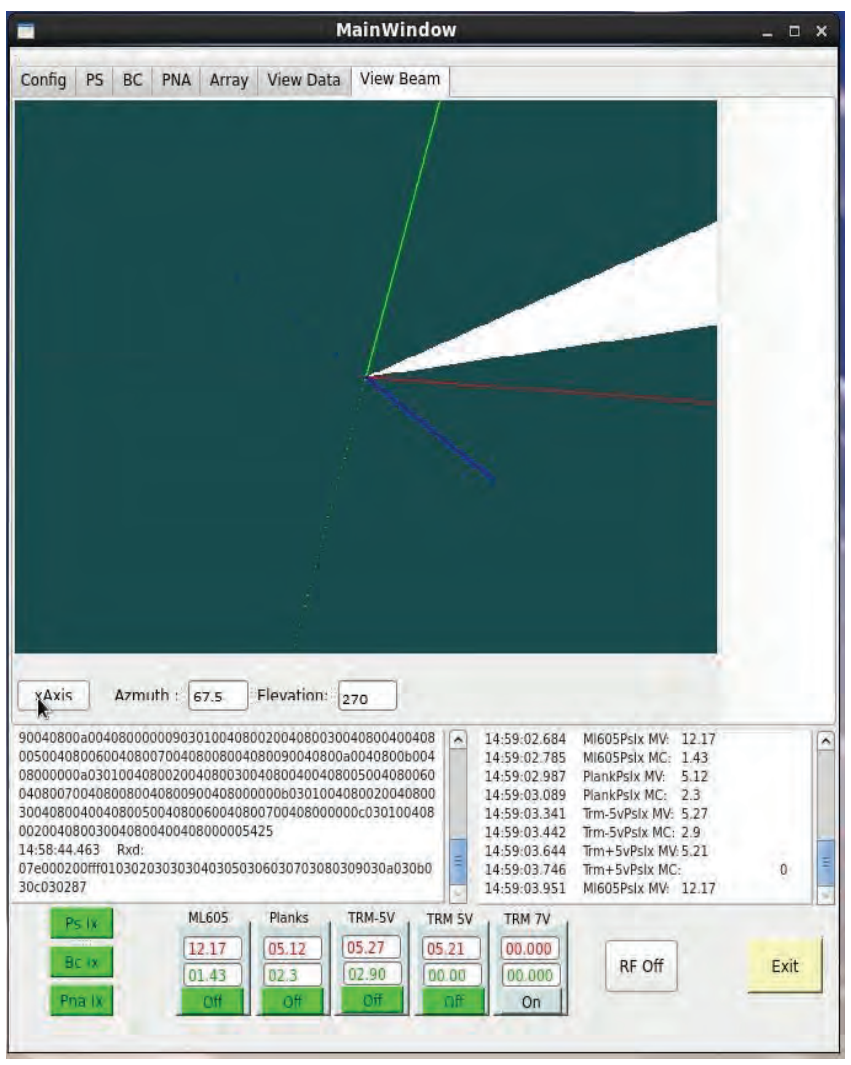

Figure 13. Beam view at certain angles

\section{F. Experimental Results of Receiver}

Based on the results, using Gnuplot software, plots are drawn. Some such plots are shown below. Through this software, 2D and 3D plots are stimulated for only receiver values of both attenuation and phase.

\section{RX Attenuation:}

The 2D plotting of Rx Attenuation values are drawn in "Fig. 14", on x-axis plane TRM no's and on y-axis plane Attenuation values with step size $5 \mathrm{db}$.

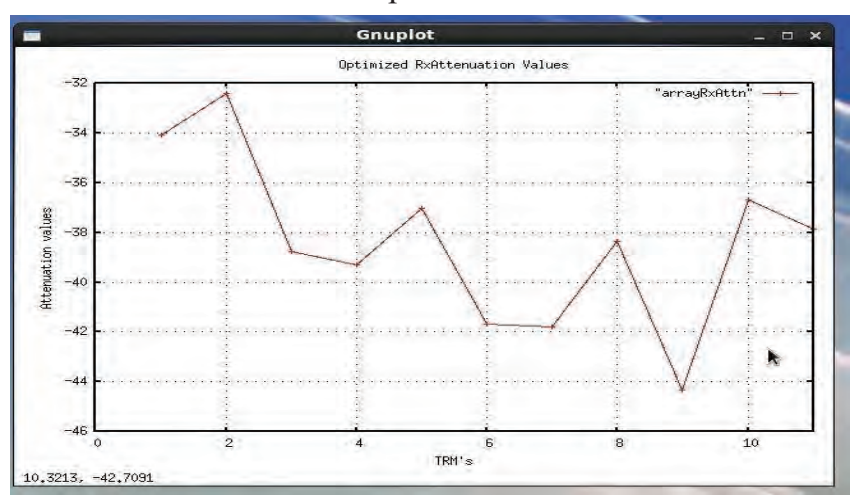

Figure 14. TRM RX Attenuation values in 2D graph

\section{RX Phase:}

The 2D plotting of Rx Phase values are shown in "Fig. 15 ", TRM no's on x-axis, and phase values (in degrees from 0 -360) on y-axis, is shown below.

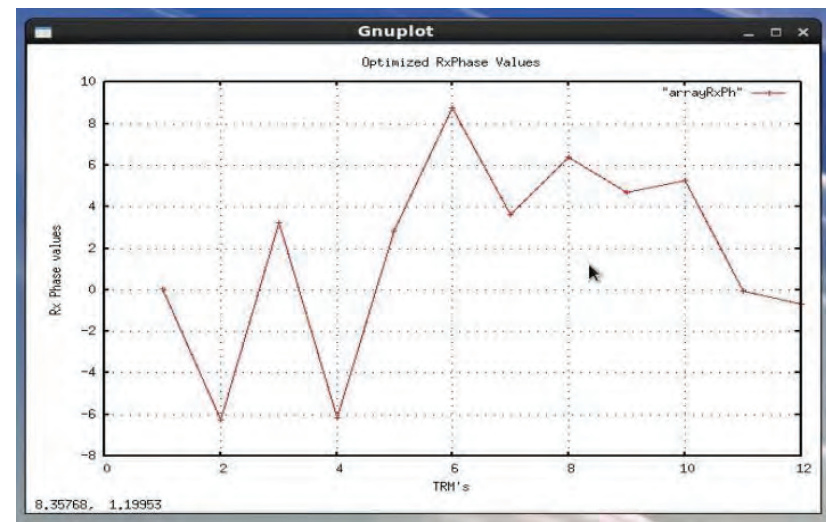

Figure 15. TRM RX Phase values in 2D graph

\section{RX Phase and Attenuation:}

The below shown "Fig.16" is the 3D plotting of both Rx phase and attenuation values.

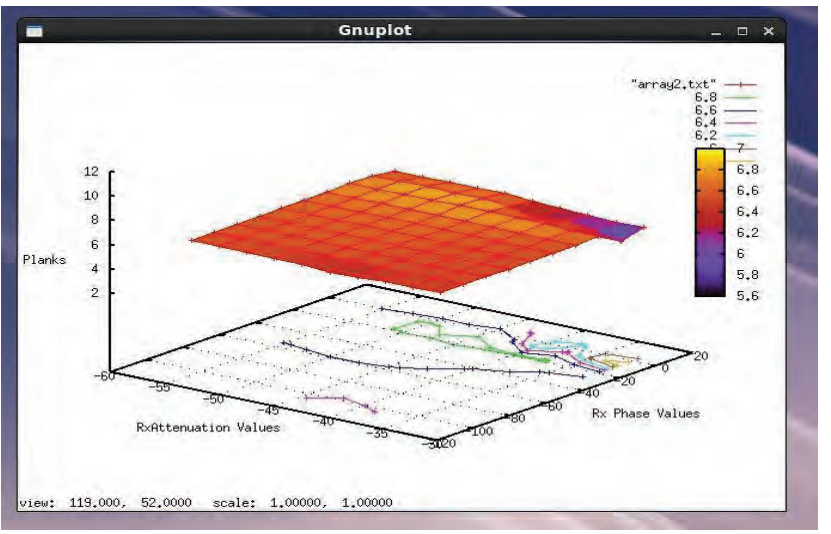

Figure 16. Both RX Phase and Attenuation values in 3D graph

\section{Conclusions}

In this paper, AESA Radar data visualization - each antenna element is controlled in such a way that the beam of radio waves can be electronically steered in different directions without moving the antenna. The beam steering is done according to the Azimuth and Elevation angles which are given through the GUI itself.

Measured results are shown in different views like file, table, tree, plotting and beam realizes the scanning range from $-60^{\circ}$ to $60^{\circ}$ in $3 \mathrm{D}$ graphics within the frequency range of operation. Using VNA (Virtual Network Analyzer) as the signal processor, the azimuth, elevation and range information are obtained by two orthogonal frequency-scanned antennas.

The further scope of the paper is application of Amplitude Tapering, which is used for measuring the amplitude values. The values are filtered in pulse mode and calibrated in $\mathrm{CW}$ mode according to the power given and based on the frequency range. According to the calibrated values beam steering is done by locking the individual targets of enemy radars. 


\section{REFERENCES}

[1] H. Wang, L. Cai, "On adaptive spatial-temporal processing for airborne surveillance radar systems", IEEE Trans. Aerosp. Electron. Syst., vol. 30, no. 3, pp. 660-669, July 1994.

[2] S. Sarns, J. Woehr, "Exploring I2C", Embedded Systems Programming.

[3] Veen, D Van and Kevin M. Buckley, Beam forming: A Versatile Approach to Spatial Filtering, IEEE ASSP Mag., April 1988.

[4] S. Kutty and D. Sen, "Beamforming for Millimeter Wave Communications: An Inclusive Survey," IEEE Commun. Surv. Tutor., vol. 18, no. 2, pp. 949-973, 2016.

[5] Li J., Jiang D. Joint Elevation and Azimuth Angles Estimation for L-Shaped Array. IEEE Antennas and Wireless Propagation Letters, vol. 16, pp. 453-456, 2017.

[6] Lee M.S. Signal modeling and analysis of a planar phasedarray FMCW radar with antenna switching. IEEE Antennas and Wireless Propagation Letters, vol. 10, pp. 179-182, 2011.

[7] Peng Z., Ran L., Li C. A K-Band Portable FMCW Radar with Beamforming Array for Short-Range Localization and VitalDoppler Targets Discrimination. IEEE Transactions on Microwave Theory and Techniques, vol, 65, no, 9, September 2017.

[8] Zrnić, D. S., and R. J. Doviak, 1976: Effective antenna pattern of scanning radars. IEEE Trans. Aerosp. Electron. Syst., 12, 551-555. 